\title{
Change detection of remotely sensed image using NDVI subtractive and classification methods.
}

\section{Israa Jameel Muhsin}

Department of Physics, College of Science, University of Baghdad, Baghdad, Iraq

E-mail: israaphsics@gmail.com

\begin{abstract}
Change detection is a technology ascertaining the changes of specific features within a certain time Interval. The use of remotely sensed image to detect changes in land use and land cover is widely preferred over other conventional survey techniques because this method is very efficient for assessing the change or degrading trends of a region. In this research two remotely sensed image of Baghdad city gathered by landsat -7 and landsat $-8 \mathrm{ETM}+$ for two time period 2000 and 2014 have been used to detect the most important changes. Registration and rectification the two original images are the first preprocessing steps was applied in this paper. Change detection using NDVI subtractive has been computed, subtractive between the bands of the two images and the ratio of the red to blue bands was also

computed. Change detection mask using minimum distance classification or detection after classification have be also used to compute the changes between the resultant classes, many statistical properties of the original and process image have been illustrated in this research
\end{abstract} Key words

Image detection,

NDVI, image

classification, image

subtractive.

\section{Article info.}

Received: Sep. 2015

Accepted: Jan. 2016

Published: Apr. 2016

\section{Sثف التغيرات للصور الفضائية باستخدام طرق حساب الفرق بين خصائص ال NDVI وطرق تصنيف الصور

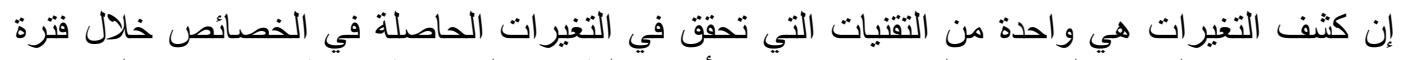

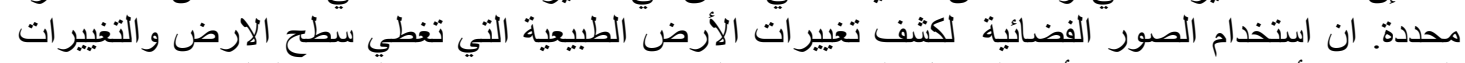

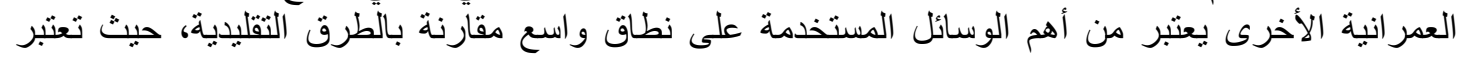

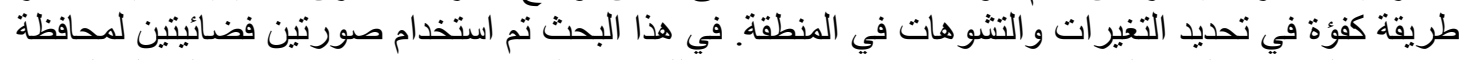

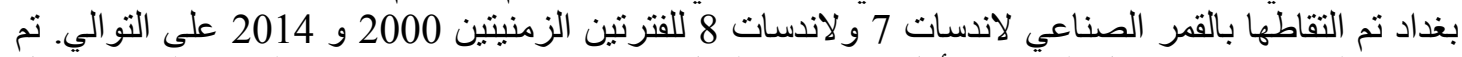



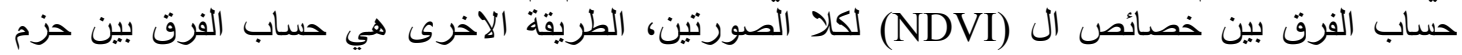



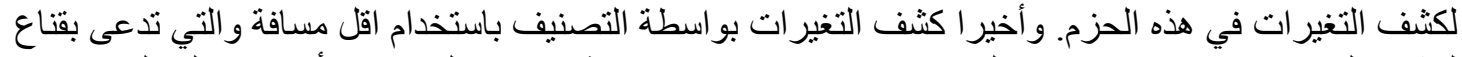



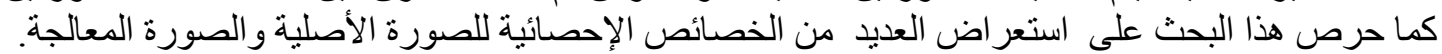

\section{Introduction}

The term of remote sensing can be defined as the science of deriving information about an object from measurements made at a distance from the object, i.e., without actually coming in contact with it [1]. Remote sensing and the spatial analysis technology have been recognized and used as powerful and effective tools to 
monitor land usage and surface changes. Satellite remote Sensing collects multi-spectrum, multi resolution, multi-period data and provides valuable information in understanding and monitoring the process of land use change, and in constructing land use databases. The main interest of this research is the change detection of land use and land cover using satellite image captured by land sat ETM+ in two different time. Digital change detection is the process that helps in determining the changes associated with land use and land cover properties with reference to georegistered multi temporal remote sensing data [2]. These land use changes can be substantial but are difficult to grasp when they occur incrementally. Recently, data from satellites has dramatically illustrated the rates at which these human-induced changes are occurring. Temporal mapping from satellite data has successfully demonstrated the utility of integrating existing historic maps with remotely sensed data and related geographic information to dynamically map land characteristics for large metropolitan areas. These regional databases provide a strong visual portrayal of recognized growth patterns, and dramatically convey how the progress of modern development results in profound changes to the landscape [3].

Change detection is a useful technology that can get temporal change information with comparisons and analysis among multi-temporal digital images [4]. There are many different techniques of change detection can be used to study the important changes which be happened in studied area for different period. This paper consist of three stages the first one is preprocessing of the original images these preprocessing include register and rectify the two images, the second stage is apply change detection techniques using subtractive of the most important characteristic of remotely sensed images such as NDVI, red to blue band ration and bands subtractive final stage is change detection mask which can be performed by classified the two images and compute the differences between the resultant classes.

\section{Studied area and data source}

Baghdad province which represents the studied area in this research located at the middle of Iraq, for more information see Fig.1 and Table 1. Geographically, its located between longitude (428955.000 m$481725.00 \mathrm{~m}) \mathrm{E}$ and latitude $(3712155.000 \mathrm{~m}-3654195.000 \mathrm{~m}) \mathrm{N}$, its consist of two part separated by the Tigris river these parts called (karkh and Riasafa). The source of the studied area images is landsat-7ETM+ for image 2000 and lansat -8 ETM+ for image 2014 with spatial resolution $30 \mathrm{~m}$ and $15 \mathrm{~m}$ for multispectral and panchromatic bands respectively.

\section{Pre-processing}

To perform the change detection process the two using images must be closely aligned. Select tie points marking the same features on both images. The program warps one image based on these tie points to match the base image. Using the ground control points dialog to select tie points is the simplest method; however, it can be challenging in areas with few obvious features, and it is time consuming. To assist tie point selection, the processing provides an automatic tie point finder tool. The tool automatically scans both images to locate common features. For best results, three to five seed points have been provide manually [5]. This co-registration is very important process must be applied on the original images before starting change detection subtractive methods. 


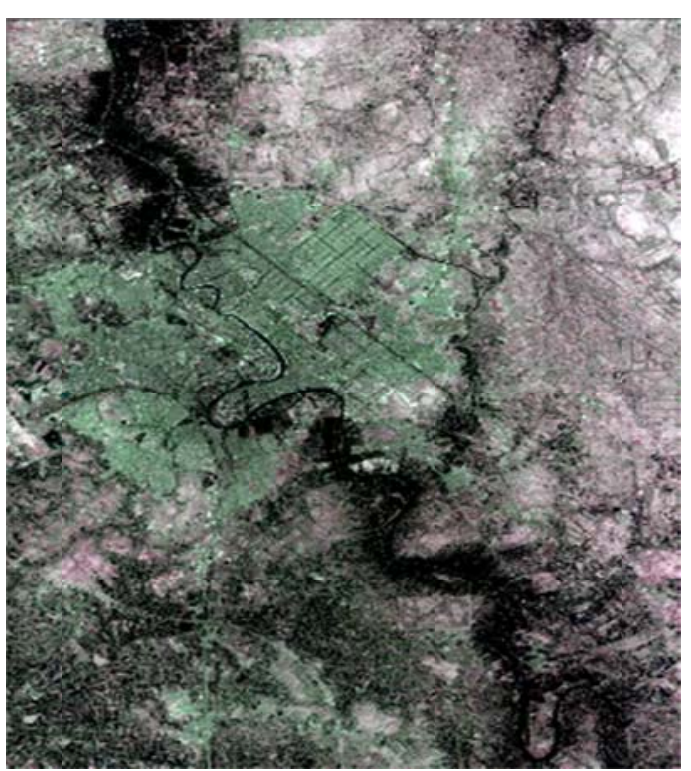

a

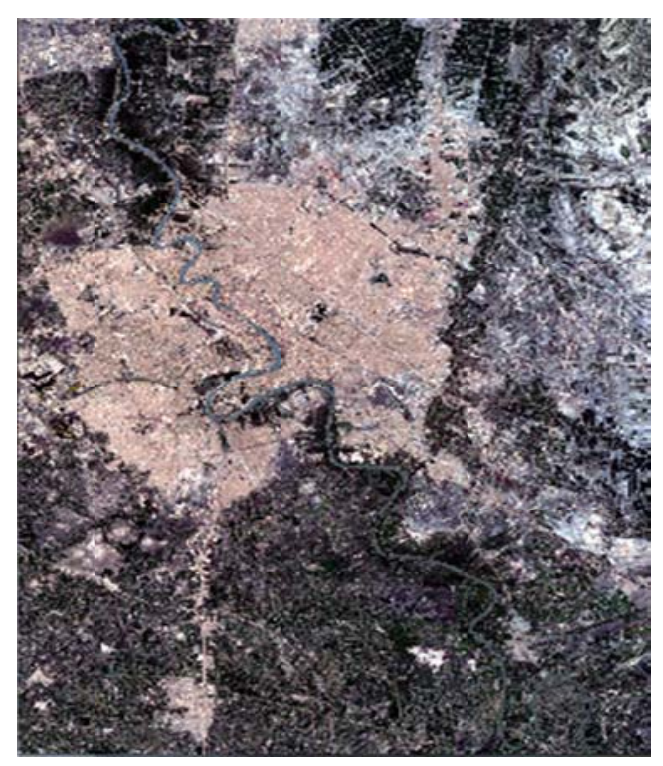

$b$

Fig. 1: a) Baghdad image captured by lansat-7 for date 2000. B) Baghdad image captured by landsat-8 for date 2014.

Table 1: Statistical properties of the two original images.

\begin{tabular}{|c|c|c|c|c|c|c|}
\hline $\begin{array}{l}\text { Images } \\
\text { date }\end{array}$ & bands & $\begin{array}{l}\text { Wavelength } \\
\qquad(\mu \mathrm{m})\end{array}$ & $\begin{array}{c}\text { Spatial } \\
\text { Resolution(m). }\end{array}$ & $\begin{array}{l}\text { Min, } \\
\text { Max }\end{array}$ & mean & Stdv. \\
\hline \multirow{4}{*}{$\begin{array}{l}\text { Baghdad } \\
\text { (2000) } \\
\text { Landsat-7 }\end{array}$} & Band1 (Blue) & $0.45-0.52$ & 30 & 0,255 & 107.55 & 54.57 \\
\hline & Band2(Green) & $0.52-0.6$ & 30 & 0,255 & 105.24 & 55.58 \\
\hline & Band3 (Red) & $0.63-0.69$ & 30 & 0,255 & 109.12 & 58.30 \\
\hline & Band4 (NIR) & $0.77-0.9$ & 30 & 0,255 & 100.69 & 56.75 \\
\hline \multirow{4}{*}{$\begin{array}{c}\text { Baghdad } \\
(2014) \\
\text { Landsat-8 }\end{array}$} & Band2(Blue) & $0.45-0.51$ & 30 & 0,255 & 102.46 & 53.34 \\
\hline & Band3(Green) & $0.53-0.59$ & 30 & 0,255 & 96.02 & 50.73 \\
\hline & Band4 (Red) & $0.64-0.67$ & 30 & 0,255 & 98.47 & 52.34 \\
\hline & Band5 (NIR) & $0.85-0.88$ & 30 & 0,255 & 143.70 & 44.93 \\
\hline
\end{tabular}

\section{Normalized Difference Vegetation Index (NDVI)}

The Normalized Difference Vegetation Index (NDVI) is an index of plant "greenness" or photosynthetic activity, and is one of the most commonly used vegetation indices. Vegetation indices are based on the observation that different surfaces reflect different types of light differently. Photo synthetically active vegetation, in particular, absorbs most of the red light that hits it while reflecting much of the near infrared light. Vegetation that is dead or stressed reflects more red lights and less near infrared light. Likewise, nonvegetated surfaces have a much more even reflectance across the light spectrum. By taking the ratio of red and near infrared bands from a remotely-sensed image, an index of vegetation "greenness" can be defined. (NDVI) is probably the most common of these ratio indices for vegetation. NDVI is calculated on a per-pixel basis as the normalized difference between the red and near infrared bands from an image [6]:

$\mathrm{NDVI}=\frac{(\mathrm{NIR}-\mathrm{RED})}{(\mathrm{NIR}+\mathrm{RED})}$

where NIR is the near infrared band value for a cell and RED is the red band value for the cell. NDVI can be calculated for any image that has a red 
and a near infrared band. The biophysical interpretation of NDVI is the fraction of absorbed photo synthetically active radiation. The NDVI was used in numerous studies estimate vegetation biomass, primary production, dominant species [6].

\section{Image subtraction method}

It is a method with the most extensive application that can be applied to a wide variety of types of images and geographical environments. It is generally conducted on the basis of gray values. The changed region and unchanged region is determined by selecting the appropriate threshold values of gray levels in the subtraction image. The gray value of the subtraction image shows the differences of corresponding pixels of two images. Its advantage is that in the subtraction image the threshold selecting operation needs to be applied only once [7]. Choosing a suitable threshold value can best separate the areas of real change and the change areas due to random factors. The gray values of the subtraction image are often approximating a Gaussian distribution, the unchanged pixels are grouped around the average value and the changed pixels are in the two tails of the distribution. In the research the second image is subtracted from the first image to provide the difference and highlight changes. The second image is more recent and the differences reflect changes over time $[8,9]$.

$$
\operatorname{Im}_{\mathrm{d}}(\mathrm{x}, \mathrm{y})=\operatorname{Im}_{1}(\mathrm{x}, \mathrm{y})-\operatorname{Im}_{2}(\mathrm{x}, \mathrm{y})
$$

where $\operatorname{Im}_{1}$ and $\operatorname{Im}_{2}$ are the images obtained from time $_{1}$ and time $2, x, y$ are the coordinates of the pixels. The resulting image $\operatorname{Im}_{d}$, represents the intensity difference of $\mathrm{Im}_{1}$ from $\mathrm{Im}_{2}$.
This technique works only if images are registered [10].

\section{NDVI difference images}

NDVI difference images easily track changes in the amount of biomass for a particular period relative to the historical average or a previous year. Thus the technique highlights areas with comparatively reduced or increased plant growth. The NDVI difference image $\left(N D V I_{d}\right)$ between two time periods is calculated by subtracting the NDVI image of the earlier time period $\left(N D V I_{1}\right)$ from the time period of interest $\left(N D V I_{2}\right)$ :

$$
N D V I_{d}=N D V I_{2}-N D V I_{1}
$$

Often average images are favored to monitor vegetation anomalies because these images normalize annual variations in plant growth. Thus, average images represent the expected growing cycle. The comparison between the year of interest and the historical average allows for the identification of actual anomalies rather than erratic changes between two single years [11]. the results of apply the subtractive method between the NDVI of the two times images can be shown in Fig. 2.

\section{Bands subtractive}

In this section the differences between the bands of the first time image $\left(\operatorname{Im}_{1}\right)$ and the bands of the second time image $\left(\operatorname{Im}_{2}\right)$ has been computed, where the first band of the first image subtractive from the first band of the second image, this subtractive was used separately for all the bands of the two images. In this way the change difference for each band has been illustrated in the below equation:

$\operatorname{Im}_{d}{ }^{k}(x, y)=\operatorname{Im}_{2}{ }^{k}(x, y)-\operatorname{Im}_{1}{ }^{k}(x, y)$ 
where: $\mathrm{k}=$ band numer, $\operatorname{Im}_{\mathrm{d}}{ }^{\mathrm{k}}$ image subtractive for each band, $(\mathrm{x}, \mathrm{y})$ pixel coordinates, $\operatorname{Im}_{1}{ }^{\mathrm{k}}$ and $\operatorname{Im}_{2}{ }^{\mathrm{k}}$ represent the first and second images. The results can be shown in Figs. 3 and 4.
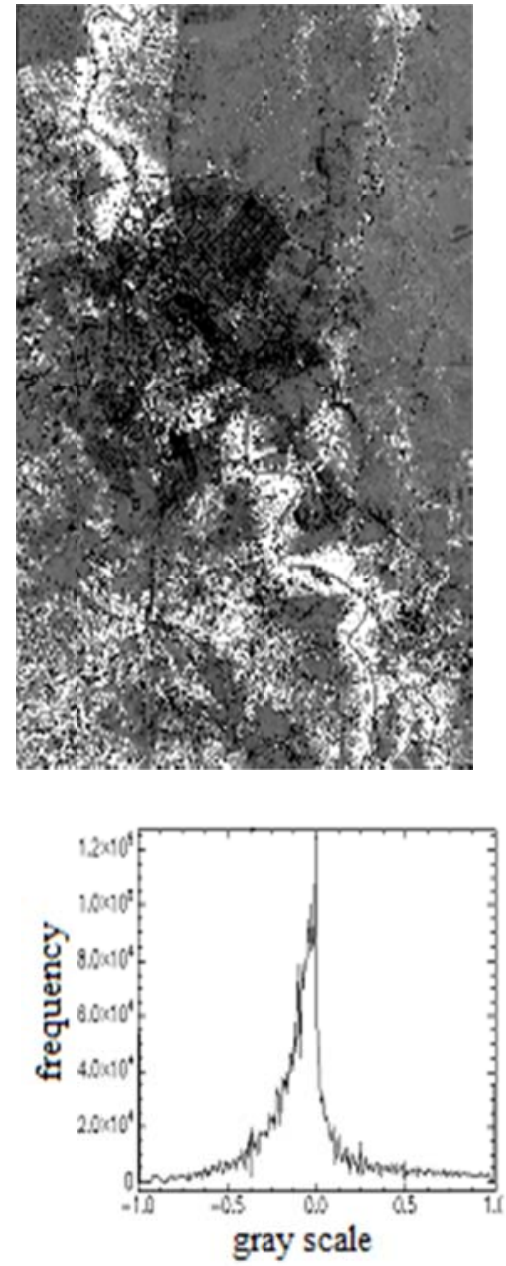

$\operatorname{NDVI}(2000)$
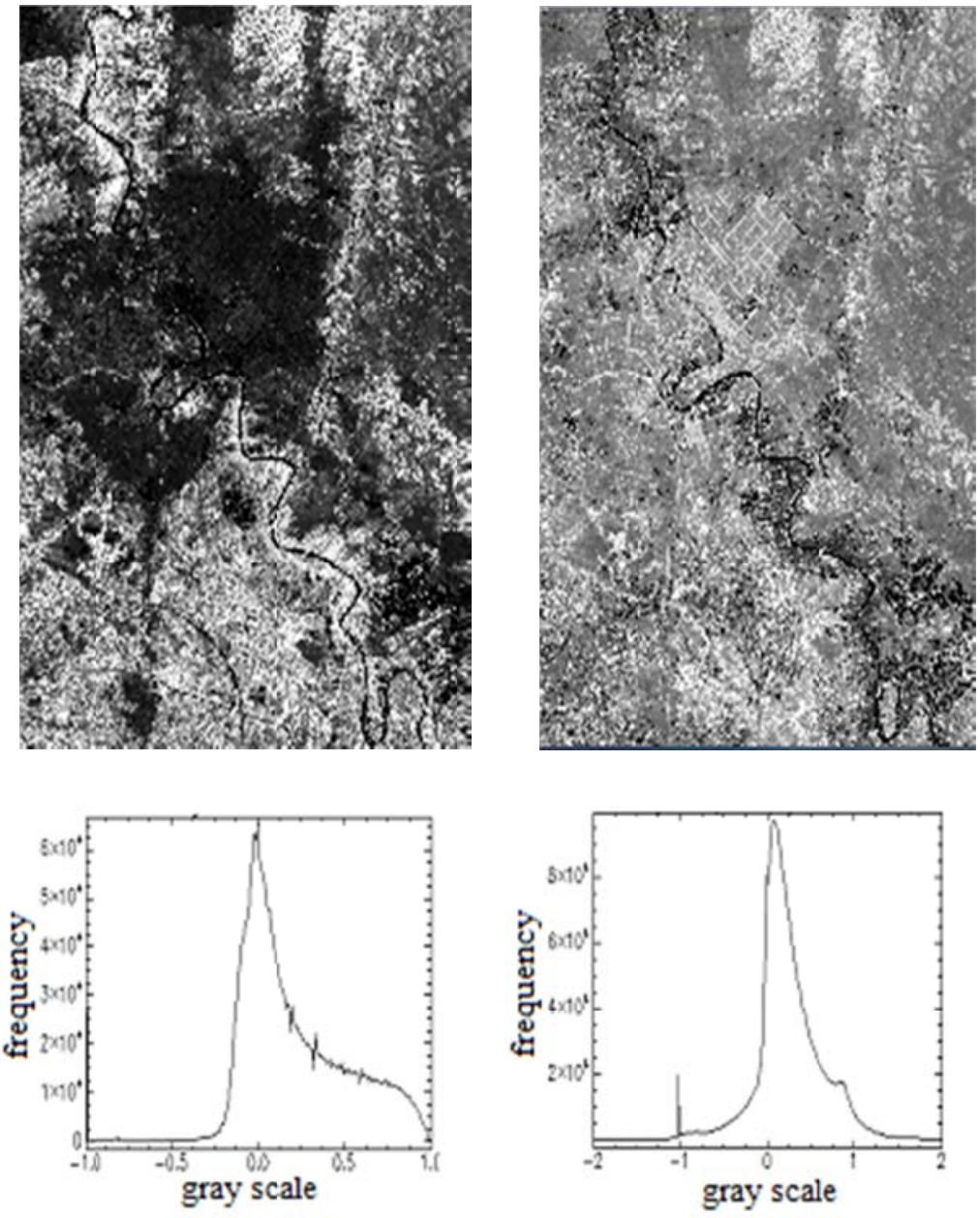

$\operatorname{NDVI}(2014)$

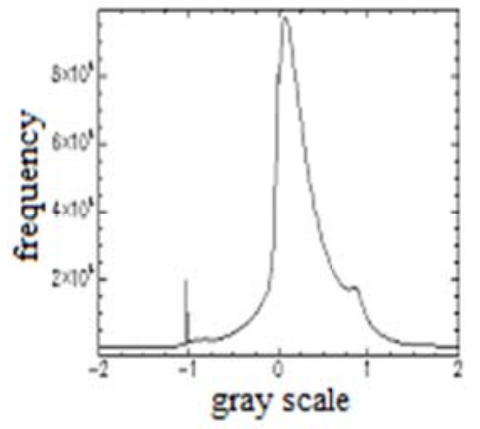

NDVI subtractive

Fig. 2: The subtractive of NDVI with histogram of the two images. 


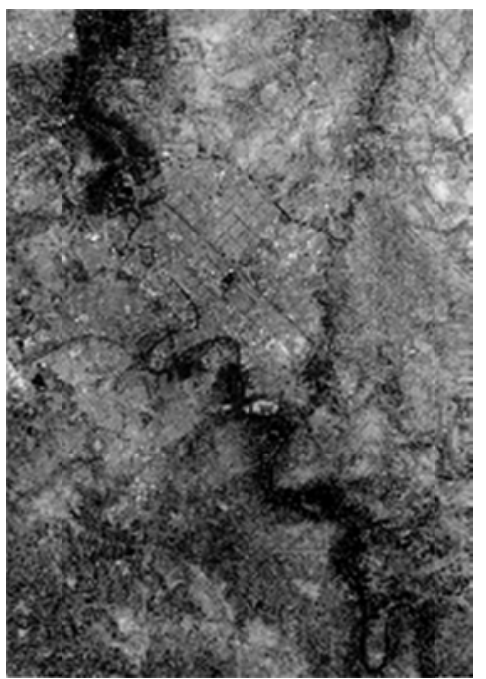

Band1(2000)

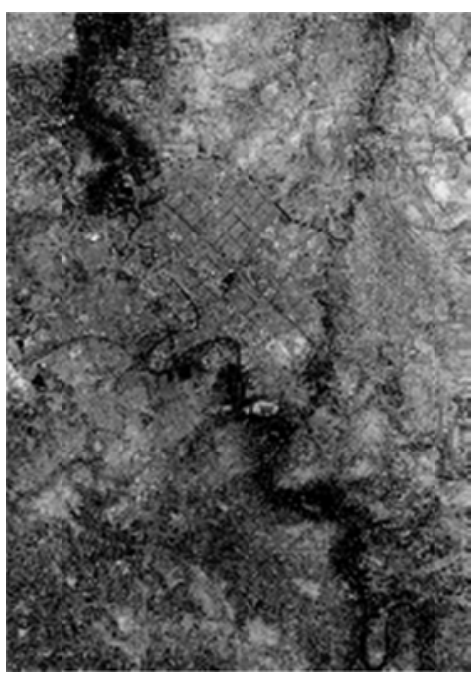

Band2(2000)



band1(2014)

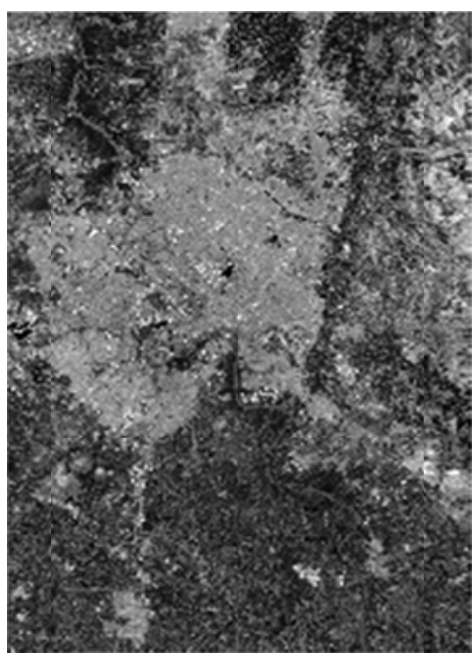

band2(2014)

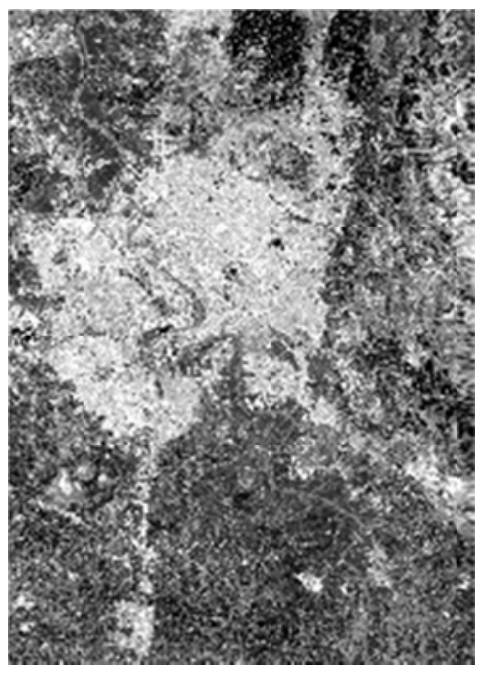

band1 subtractive

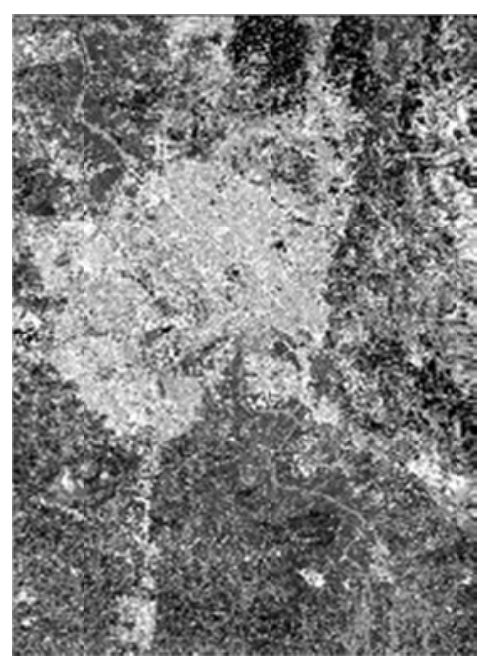

band 2 subtractive

Fig. 3: The subtractive of first and second bands of the two images. 


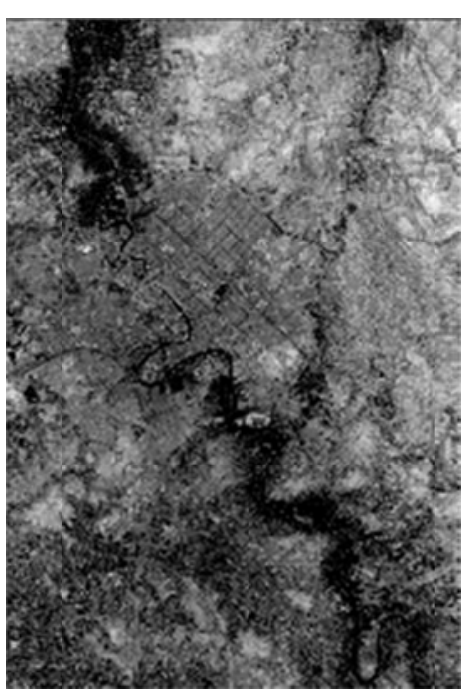

Band3(2000)

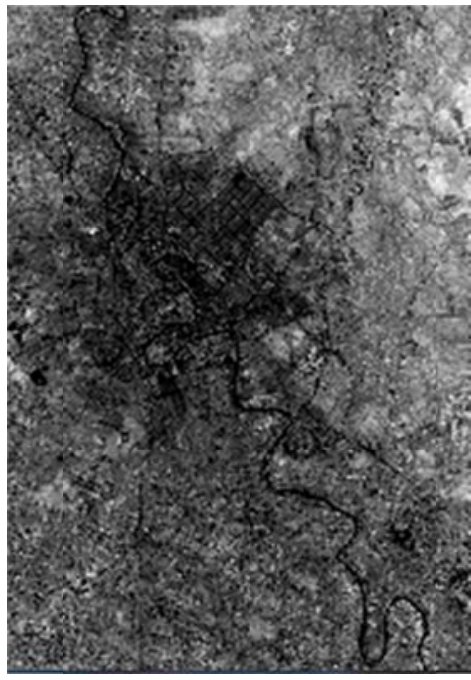

Band4(2000)

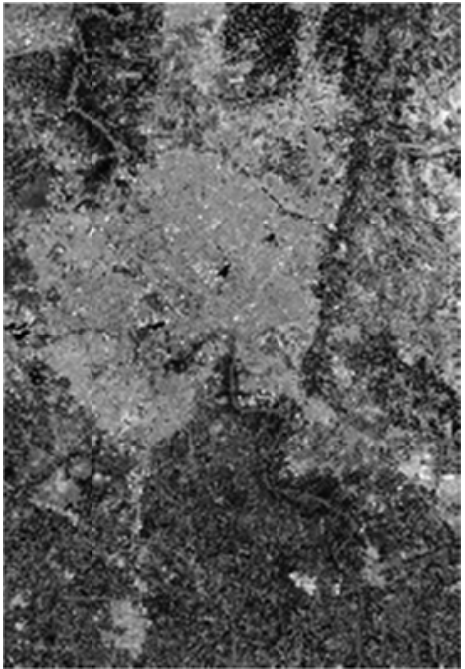

band3(2014)

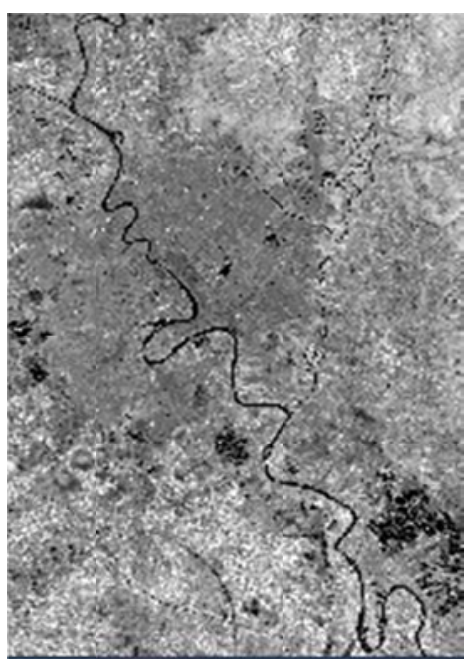

band4(2014)

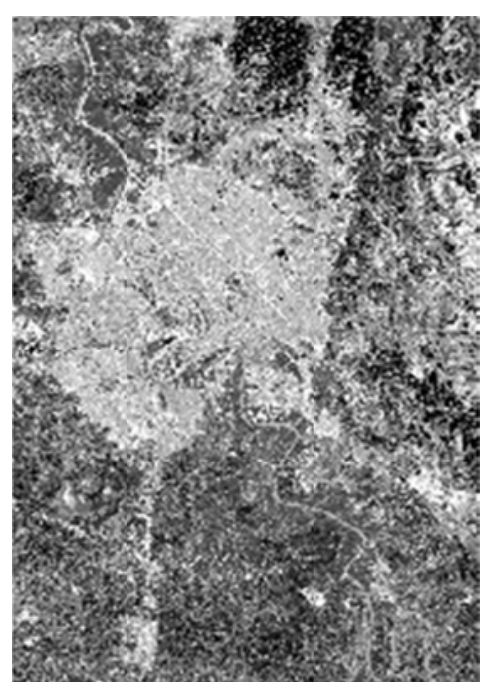

band3 subtractive



band4 subtractive

Fig. 4: The subtractive of third and fourth bands of the two images.

\section{Red to blue bands ratio subtractive}

In this section the ratio of the red to blue bands of each image have been computed, then the subtractive between these ratio was calculated to illustrate the changes between the two images, the mathematic difference has been shown in Eq.(5).
$D\left(I M_{r}\right)=\frac{R\left(I M_{2}\right)}{B\left(I M_{2}\right)}-\frac{R\left(I M_{1}\right)}{B\left(I M_{1}\right)}$

where: $D\left(I M_{r}\right)$ is the difference image, $R\left(I M_{1}\right)$ and $B\left(I M_{1}\right)$ is red and blue bands of the first image, $R\left(I M_{2}\right)$ and $B\left(I M_{2}\right)$ is red and blue bands of the second image. The results of apply red to blue ratio subtractive between the two images can be shown in Fig.5. 


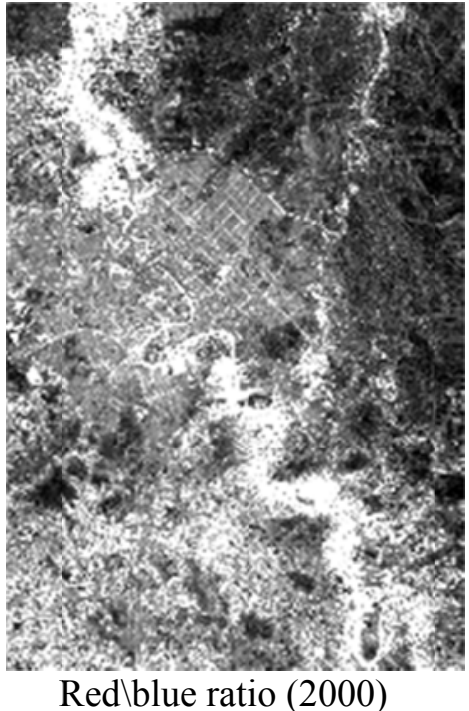

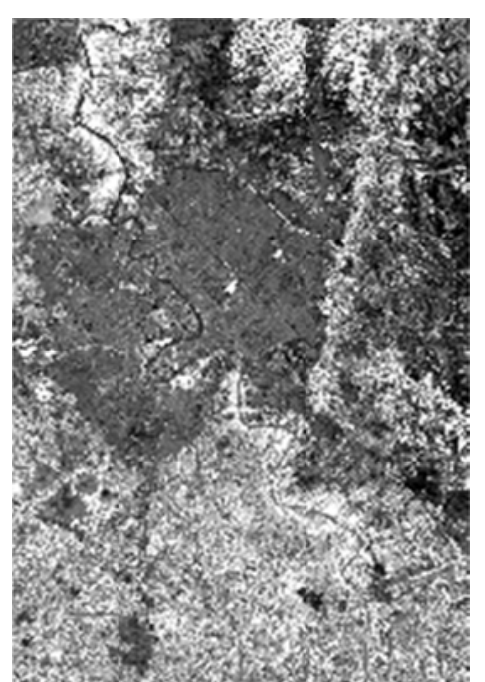

Red $\backslash$ blue ratio (2014)

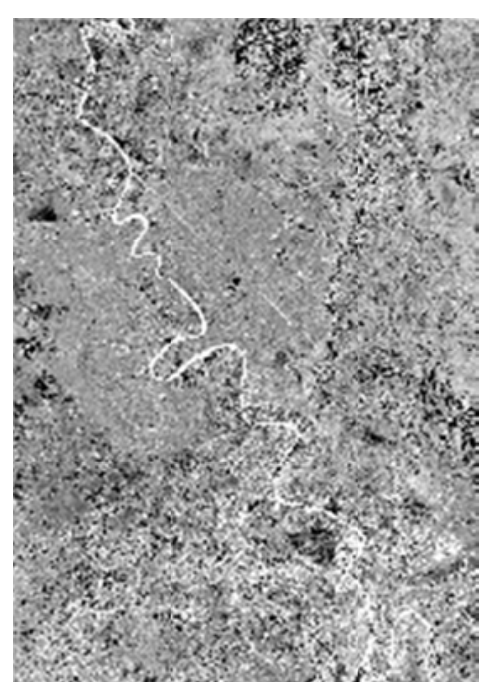

Red $\backslash$ blue ratio subtractive

Fig. 5: The subtractive of Red to Blue bands of the two images.

\section{Change detection mask using supervised classification}

The method of change detection after classification is the most simple change detection analysis techniques based on the classification. After classification compare method can be used to two or more images after registration, including a classification step and a comparing step. Each image of multi-temporal images is classified separately and then the classification result images are compared. If the corresponding pixels have the same category label, the pixel has not been changed, or else the pixel has been changed [7]. In this research the supervised classification using minimum distance classifier has been used to classify the two time images.

The discriminate function for the minimum distance classifier is developed as follows. Suppose $m_{i}, i=$ $1, \ldots M$ are the means of the $M$ classes determined from training data, and $x$ is the position of the pixel to be classified. Compute the set of squared Euclidean distances of the unknown pixel to each of the class means, defined in vector form as

$d\left(x, m_{i}\right)^{2}=\left(x-m_{i}\right)^{t}\left(x-m_{i}\right)=(x-$ $\left.m_{i}\right) .\left(x-m_{i}\right), i=1, \ldots M$
Expanding the product gives

$$
d\left(x, m_{i}\right)^{2}=x \cdot x-2 m_{i} \cdot x+m_{i} \cdot m_{i}(7)
$$

Classification is performed on the basis of

$x \in \omega_{i}$ if $d\left(x, m_{i}\right)^{2}<d\left(x, m_{j}\right)^{2}$ for all $j \neq i$

Note that $x \cdot x$ is common to all $d\left(x, m_{j}\right)^{2}$ and thus can be removed. Moreover, rather than classifying according to the smallest of the remaining expressions, the signs can be reversed and classification performed on the basis of

$x \in \omega_{i}$ if $g_{i}(x)>g_{j}(x)$ for all $j \neq i$

where

$g_{i}(x)=2 m_{i} \cdot x-m_{i} \cdot m_{i}$, etc.

Eq.(10) defines the discriminate function for the minimum distance classifier [12]. The results of classified image for five classes can be shown in Fig.6 and the results of subtractive between each class of each image can be illustrated in Fig. 7. While Table 2 illustrates the statistical distribution of the classes after classification. 




Classified image (2000)

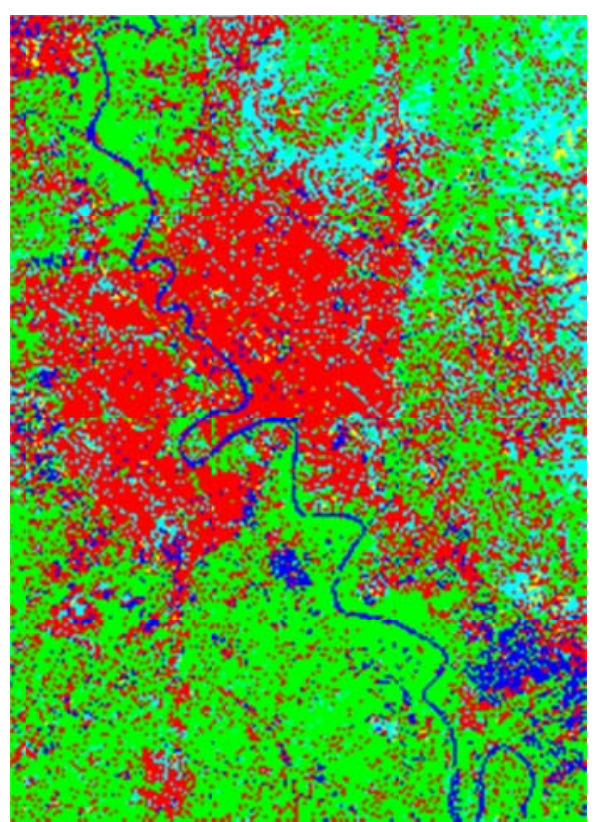

Classified image (2014)

Fig. 6: The classified images using minimum distance classification.

Table 2 : The statistiacal distribution of the classified image using minimum distance classifier.

\begin{tabular}{|ccccc|}
\hline \multirow{4}{*}{ Image } & Classes & Bits No. & Total & Percent $\%$ \\
\cline { 2 - 5 } Image 2000 & buldings & 876376 & 876376 & 25.7600 \\
\cline { 2 - 5 } & vegatation & 722129 & 1598505 & 21.2261 \\
\cline { 2 - 5 } & water & 103130 & 1701635 & 3.0314 \\
\cline { 2 - 5 } & Soil & 289122 & 1990757 & 8.4984 \\
\cline { 2 - 5 } Image 2014 & roads & 1411323 & 3402080 & 41.4841 \\
\cline { 2 - 5 } & buldings & 898515 & 898515 & 26.4108 \\
\cline { 2 - 5 } & vegatation & 1352277 & 2250792 & 39.7485 \\
\cline { 2 - 5 } & water & 225882 & 2476674 & 6.6395 \\
\cline { 2 - 5 } & Soil & 124193 & 2600876 & 3.6505 \\
\cline { 2 - 5 } & roads & 801213 & 3402080 & 23.5507 \\
\hline
\end{tabular}




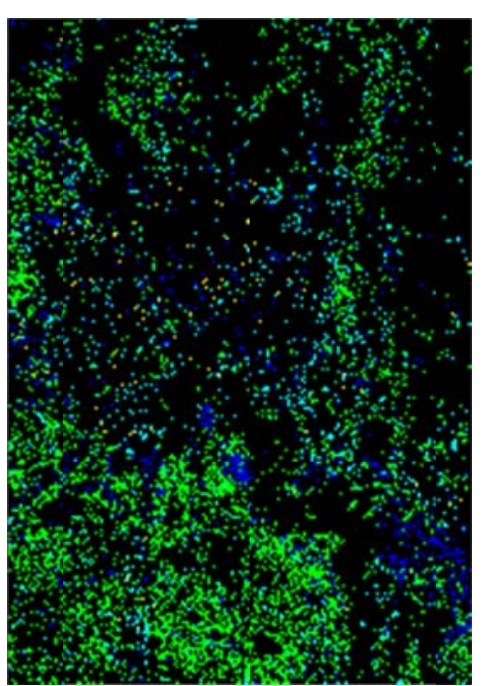

Class (1) difference

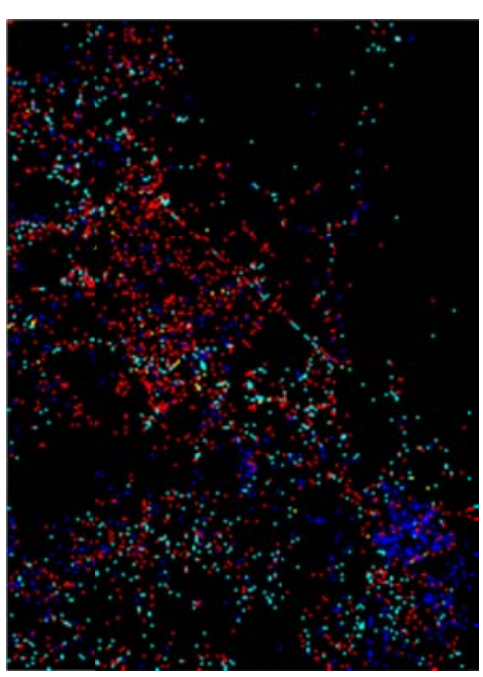

Class (2) difference

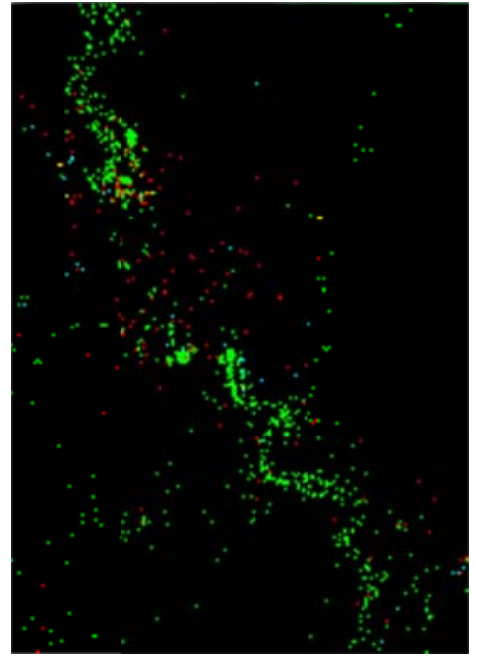

Class(3) difference

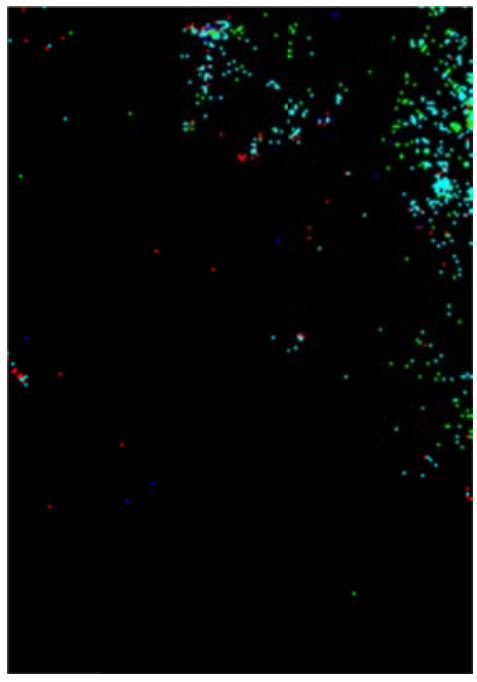

Class (4) difference

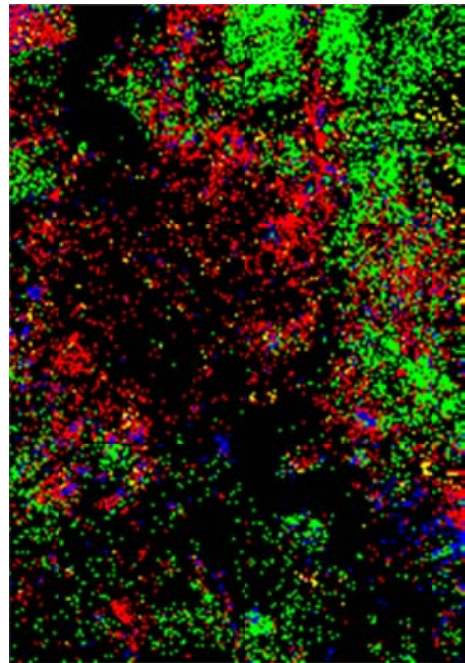

Class (5) difference

Fig.7: The change detection mask between the classes of the two classified image using minimum distance classifier.

\section{Statistical properties}

The statistics properties of Tables 3-5 list the image 2000 (initial state) classes in the columns and the image 2014 (final state) classes in the rows. However, the columns include only the selected initial state classes, while the rows contain all of the final state classes. The class total row indicates the total number of pixels in each initial state class, and the class total column indicates the total number of pixels in each final state class. The row total column is a class-by-class summation of all final state pixels that fell into the selected initial state classes. The class changes row indicates the total number of initial state pixels that changed classes. The image difference row is the difference in the total number of equivalently classed pixels in the two images, computed by subtracting the initial state class totals from the final state class totals. An image difference that is positive indicates that the class size increased [5]. In the bellow tables, it three types of data are shown, Table 1 present the data as pixel values, while Table 2 show the area of classes with $\mathrm{km}$ unit, finally the percentage of each 
class is shown and can be computed using the following equation:
Percentage $=($ final state - initial state $) /$ initial state.

Table 3: The statistic properties of the initial and final classes using pixel unit.

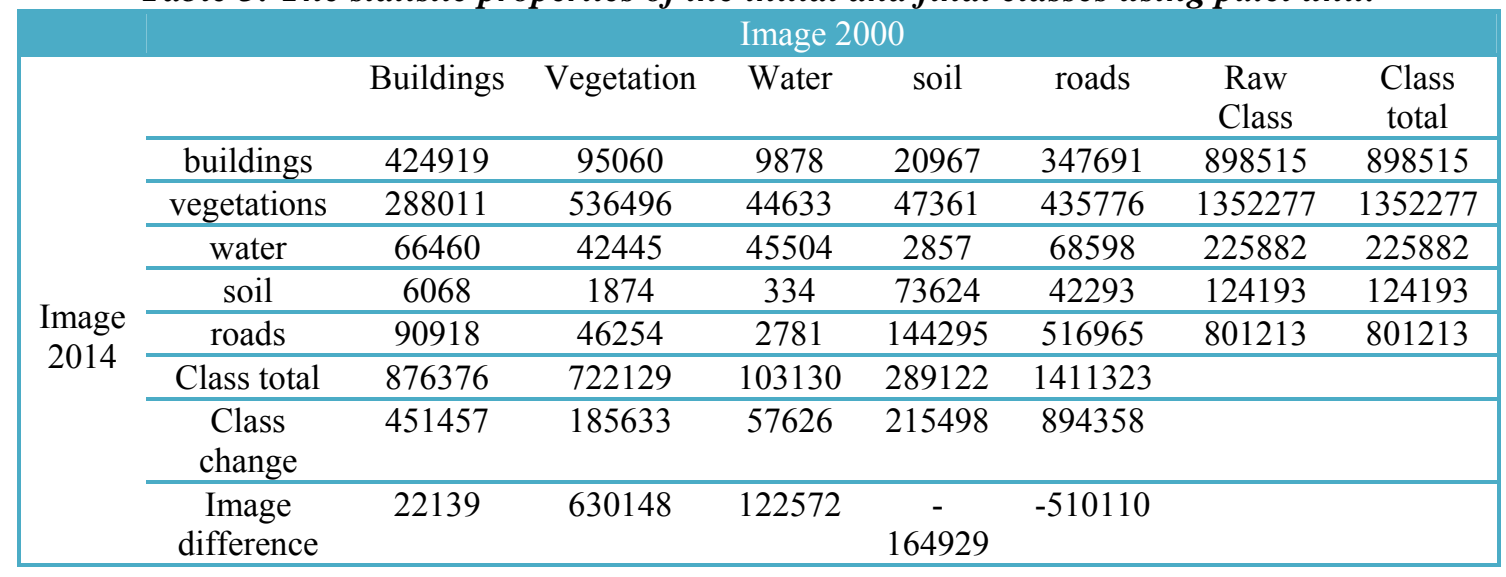

Table 4: The area of the initial and final classes using kilometer unit.

\begin{tabular}{|c|c|c|c|c|c|c|c|c|}
\hline & & & & Image 2 & & & & \\
\hline & & Buildings & Vegetation & Water & soil & roads & $\begin{array}{l}\text { Raw } \\
\text { Class }\end{array}$ & $\begin{array}{l}\text { Class } \\
\text { total }\end{array}$ \\
\hline & buildings & 382.43 & 85.55 & 8.89 & 18.87 & 312.92 & 808.66 & 808.66 \\
\hline & vegetations & 259.21 & 482.85 & 40.17 & 42.62 & 392.20 & 1217.05 & 1217.05 \\
\hline & water & 59.81 & 38.20 & 40.95 & 2.59 & 61.74 & 203.29 & 203.29 \\
\hline & soil & 5.46 & 1.69 & 0.30 & 66.26 & 38.06 & 111.77 & 111.77 \\
\hline Image & roads & 81.83 & 41.63 & 2.50 & 129.87 & 465.27 & 721.09 & 721.09 \\
\hline & Class total & 788.74 & 649.92 & 92.82 & 260.21 & 1270.19 & & \\
\hline & $\begin{array}{l}\text { Class } \\
\text { change }\end{array}$ & 406.31 & 167.07 & 51.86 & 193.95 & 804.92 & & \\
\hline & $\begin{array}{c}\text { Image } \\
\text { difference }\end{array}$ & 19.93 & 567.13 & 110.48 & -148.44 & -549.10 & & \\
\hline
\end{tabular}

Table 5: The statistic properties of the initial and final classes using percent form.

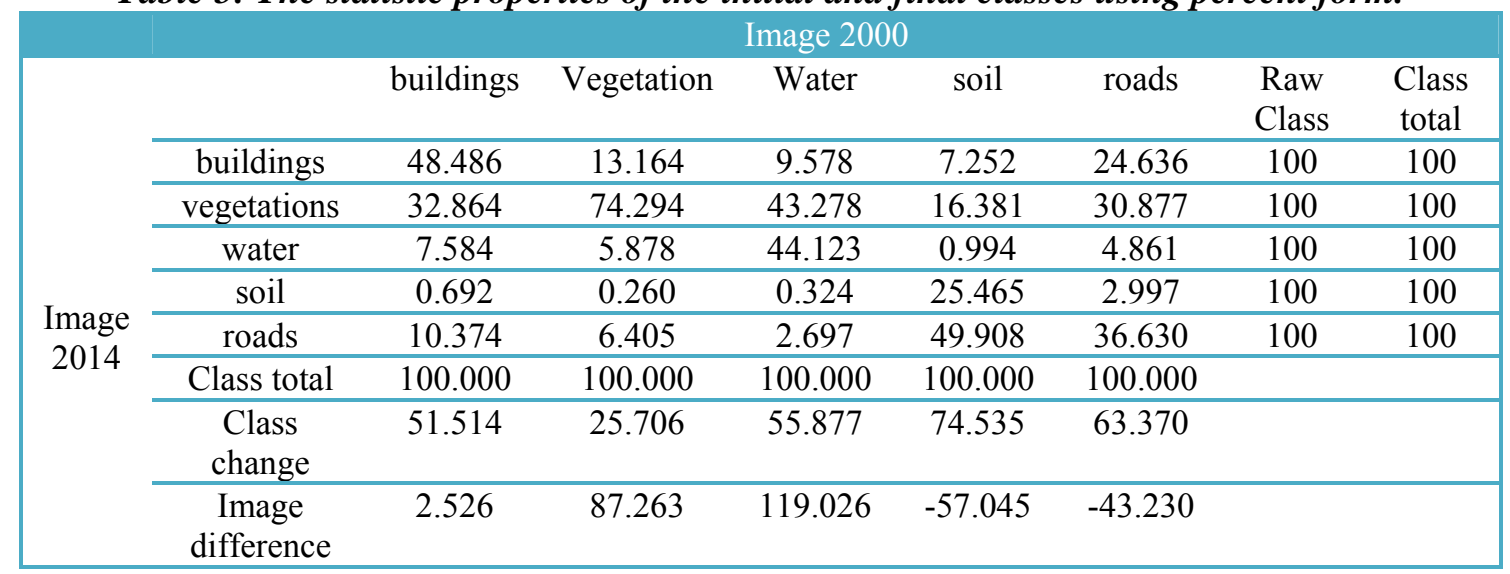

\section{Discussion and conclusions}

The Change Detection analysis is an efficient method of studying the changes observed in the studied area. There are many techniques have been used to detect the change in the land use and land cover such as building, vegetation, water, soil, roads. These techniques dependent on the subtractive way between the characteristics of the using images. The difference between the landsat-7 image captured in 2000 and landsat- 8 image captured in 2014 have been 
achieved by the computed the NDVI of each image and the subtractive between the NDVI of them. It is used to distinguish healthy vegetation from others or from non-vegetated areas and it is consider being the scale of vegetation. The result of NDVI images and NDVI histogram in Fig. 2 show that increase of pixels ranged from 0.2 to 0.8 which represent the green values in 2014 compared with 2000 belong to interest of local government of culture and cover most of Baghdad free areas with grass, in other hand the increase is not clear and may include in addition to the green vegetation area areas of wet and dry vegetation also. The increasing of vegetation areas between time 2000 and 2014 is not much because convert most of farms of Baghdad city to buildings, small increase in agricultural areas accompanied by a slight increase in expansion of water regions. image subtraction method such as bands subtraction and red to blue ratio subtraction reduces the probability of errors. The background gray is restrained and the subtraction gray is enhanced in image subtraction processing. It is benefit for picking-up the information: such as water and underwater channels ditch. Image ratio method is useful for the extraction of vegetation and texture. However, there is still some changes information not being detected such as the road and the circular feature in the central. the image subtraction and image ratio methods are capable only of detecting the position and degree of changes but cannot provide information about the type of changes, such as what class was changed to what, which can be provided by change detection after classification. But the accuracy of change detection after classification would be affected greatly and may be not fit for change detection of the details of urban because of the low- resolution $(30 \mathrm{~m})$ of multi-spectral images and complex spectral characteristics of the cities. The change of each class is shown in Fig. 7 where each class of image 2000 subtractive from the class of image 2014. To increase the accuracy of change detection mask or change detection after classification is applied must be used high spatial resolution image such as quick bird $(0.6 \mathrm{~m}-4 \mathrm{~m})$ and word view-2(0.5m-2m) land sat. on the other hand the supervised classification which be used in this research depend on the user skilled of selecting the regions of interest.

\section{References}

[1] P. H.Swain and S. M Davis. "Remote Sensing: The Quantitative Approach" 1st Edition, New York: McGraw-Hill, pp 396 , 1978

[2] Yacouba Diallo1, Guangdao Hu1, Xingping, Yunnan Province, Journal of American Science 5, 4 (2009) 157-166. [3] James Bolarinwa Olaleye, Oludayo Emmanuel Abiodun and Queen Igbokwe, "Land Use Change Detection and Analysis Using Remotely Sensed Data in Lekki Peninsula Area of Lagos, Nigeria'. TS 8B - SIM in Planning and Development, FIG Working Week, (2009) 1-15.

[4] Ayman R. EL Shehaby, Hossam H. AL Semary, Mahmoud Salah, Shaimaa M. Ibrahim, Journal of Civil Engineering Research, 2, 6 (2012) 5763.

[5] ENVI 4.5 Guide (2008).

[6] Zuzana Maskova, Frantisek Zemek, Jan Kvet., Borel Enviroment Research 13, (2008) 417-432.

[7] Zhang Shaoqing, $\mathrm{Xu} \mathrm{Lu,} \mathrm{The}$ International Archives of the Photogrammetry, Remote Sensing and Spatial Information Sciences. Vol. XXXVII. Part B7. Beijing 2008, 15951598. 
[8] F. Fung, E. Le Drew, Photogrammetry and Remote Sensing Photogrammetric Engineering and 80 (2013) 91-106

Remote Sensing, 53, 12 (1987) 1649-

[11] Dorit Gross "Monitoring 1658.

[9] Wang Fangju, IEEE Trans Agricultural Biomass Using NDVI Time Series", Food and Agriculture Geoscience and Remote Sensing, 31, 1 (1993) 36-145.

[10] Masroor Hussain, DongmeiChen, AngelaCheng, HuiWei, DavidStanley, ELSEVIAR, ISPRS Journal of Organization of the United Nations (FAO) Rome, (2005).

[12] J.A. Richards, Remote Sensing Digital Image Analysis Springer-Verlag, Berlin,(1999) p. 240. 